\title{
Condorcet consistent scoring rules and single-peakedness
}

\author{
by \\ Dolors Berga \\ Departament d'Economia, Universitat de Girona, C/ Universitat de Girona, \\ 10, 17003, Girona, Spain. E-mail: dolors.berga@udg.edu \\ Guadalupe Correa-Lopera* \\ PhD Program in Economics and Business, Universidad de Málaga, Spain. \\ PhD Program in Economics and Management, Università degli Studi di \\ Padova, Italy. Universidad de Málaga, Campus El Ejido, 29071 Málaga, Spain. \\ E-mail: guadalupecorrealopera@gmail.com \\ and \\ Bernardo Moreno \\ Departamento de Teoría e Historia Económica, Universidad de Málaga, \\ Campus El Ejido, 29071 Málaga, Spain. E-mail: bernardo@uma.es
}

May 15th, 2019

\begin{abstract}
We study voting problems with an odd number of agents and single-peaked preferences. With only three alternatives, there are scoring rules that yield the Condorcet winner only for committees of three and five agents. With four or more alternatives, only committees of three agents work. In all these scoring rules, the best and worst alternatives are assigned a score of 1 and 0 , respectively, and any middle alternative a score between 0 and $\frac{1}{2}$. For five or more alternatives, the score of any middle alternative must be the same, and we call this family semiplurality scoring rules.
\end{abstract}

Keywords: Scoring rule, Condorcet winner, single-peakedness, semiplurality rule.

Journal of Economic Literature Classification Number: D71, D72.

*Corresponding author: E-mail: guadalupecorrealopera@gmail.com; Postal Address: Universidad de Málaga, Campus El Ejido, 29071 Málaga, Spain 


\section{Introduction}

When only two alternatives are at stake, majority voting is the best method, the one satisfying well-known properties (see May, 1952). The challenge is to extend majority voting among pairs to situations where there are more than two alternatives. Condorcet proposed choosing the alternative defeating every other alternative in pairwise comparisons (Condorcet, 1785). Borda proposed assigning points to each candidate according to preferences and choosing the alternative with the highest total score (Borda, 1781). These two proposals lead to the two most acknowledged families of voting rules, Condorcet winner rules and scoring (or positional) rules (Smith, 1973; Young, 1975). The Condorcet consistent criterion, requiring the election of the Condorcet winner when it exists, is a widely used principle for evaluating alternative voting rules. On an unrestricted domain of preferences, for any odd number of voters and any number of alternatives higher than two, each scoring rule is not Condorcet consistent for some preference profile (Fishburn, 1973). In this paper we consider the set of single-peaked preferences and we are interested in analyzing which scoring rules, if any, are Condorcet consistent.

Finding out what is the "optimal" size of a group of voters has been paid attention in the literature. In the context of the Condorcet's jury theorem, where a group of voters wants to reach the "correct" decision by majority voting, the number of voters that should be included in the group depends on the probability of voting for the correct decision of each voter (Condorcet, 1875; Grofman, Owen, and Feld, 1983).

While Section 2 presents the model, Section 3 presents the main results and their proofs. Other results are placed in the Appendix.

\section{Model}

Let $A=\left\{a_{1}, a_{2}, \ldots, a_{m}\right\}$ be a finite set of $m \geq 3$ alternatives where $a_{1}<a_{2}<$ $\ldots<a_{m}$, and $N=\{1, \ldots, n\}$ be an odd finite set of $n \geq 3$ agents. Each agent $i$ 's preferences are linear orders over alternatives, denoted by $R_{i}$, which are single-peaked on $A$. For any $a_{l}, a_{k} \in A, a_{l} R_{i} a_{k}$ denotes that $a_{l}$ is strictly preferred to $a_{k}$ by $i$ at $R_{i}$. A preference $R$ is single-peaked on $A$ if (i) there exists an alternative $p(R)$, the peak of $R$, such that $p(R) R a_{l}$ for any $a_{l} \in A$, and (ii) for every pair of alternatives $a_{l}, a_{k} \in A$ such that $p(R)<a_{l}<a_{k}$ or $a_{k}<a_{l}<p(R), a_{l} R a_{k}$. Let $\mathcal{S}$ denote the set of all single-peaked preferences on $A$. Elements in $\mathcal{S}^{n}$ are called preference profiles, and are denoted by $R_{N}=$ $\left(R_{1}, \ldots, R_{n}\right)$. A voting rule $r: \mathcal{S}^{n} \rightarrow 2^{A} \backslash \varnothing$ selects a non-empty set of alternatives for each preference profile. We define two types of voting rules: the Condorcet winner rule and the scoring rules.

We say that an alternative $a_{j}$ defeats alternative $a_{k}$ in majority comparison if a strict majority of agents prefers $a_{j}$ over $a_{k}$. When $n$ is odd and preferences are single-peaked, there always exists a unique alternative that defeats any other by majority comparisons, which is called the Condorcet winner. The Condorcet 
winner rule $C W$ assigns to each preference profile the Condorcet winner, that is, the unique alternative that defeats every other alternative in pairwise majority comparisons.

Fix a vector $w=\left(w_{0}, \ldots, w_{m-1}\right) \in \mathbb{R}^{m}$ satisfying $w_{0} \leq w_{1} \leq \ldots \leq w_{m-2} \leq$ $w_{m-1}$ and $w_{0}<w_{m-1}$. Without loss of generality, we assume that $w_{0}=0$ and $w_{m-1}=1$. Points are assigned to every alternative in such a way that if alternative $x$ is in the $j$ 'th top position according to $R_{i}$, then $x$ receives $s_{w}\left(x, R_{i}\right)=w_{m-j}$ points from agent $i$. Given a preference profile $R_{N}$ and an alternative $x$, let $s_{w}\left(x, R_{N}\right)=\sum_{i=1}^{n} s_{w}\left(x, R_{i}\right)$ be the score assigned to $x$ at $R_{N}$. The $w$-scoring rule $S_{w}$ assigns to each preference profile the set of alternatives with the highest score. ${ }^{1}$

Given $A$ and $N$, we say that a $w$-scoring rule is Condorcet consistent if for any $R_{N} \in \mathcal{S}^{n}, C W\left(R_{N}\right) \in S_{w}\left(R_{N}\right)$.

For each $m$, call semiplurality rules the family of $w$-scoring rules where $w_{m-2}=w_{1} \in\left[0, \frac{1}{2}\right]$. Note that plurality is a member of this family for $w_{1}=0$. The Borda count for $m$ alternatives is the $w$-scoring rule where $\left|w_{l}-w_{l+1}\right|=\left|w_{l+1}-w_{l+2}\right|$ for all $l=0, \ldots, m-2$. The Borda count is a semiplurality rule only for $m=3$ and $w_{1}=\frac{1}{2}$.

\section{Results and Discussion}

For each size of the society and the set of alternatives, we characterize the subset of $w$-scoring rules that are Condorcet consistent. We focus on the cases of three or more alternatives because in the case of two alternatives each $w$-scoring rule trivially chooses the Condorcet winner. We obtain positive results for specific situations, but only with three or five agents. The characterization is obtained in Propositions 1, 2, and 3.

Proposition 1 Let $n=3$. (i) For $m \neq 4$, a w-scoring rule is Condorcet consistent if and only if $w_{m-2} \in\left[0, \frac{1}{2}\right]$ and $w_{m-2}=w_{1}$. (ii) For $m=4$, a $w$-scoring rule is Condorcet consistent if and only if $w_{m-2} \in\left[0, \frac{1}{2}\right]$. $\left[0, \frac{1}{2}\right]$.

For $m \neq 4$, this is the family of semiplurality scoring rules with $w_{m-2} \in$

Proposition 2 Let $n=5$. (i) For $m=3$, a w-scoring rule is Condorcet consistent if and only if $w_{m-2}=\frac{1}{3}$. (ii) For $m \geq 4$, no w-scoring rule is Condorcet consistent.

Proposition 3 For $n>5$ and any $m \geq 3$, no $w$-scoring rule is Condorcet consistent.

\footnotetext{
${ }^{1}$ Our definition of scoring rules is from Moulin (1988). See Young (1975) and also Bossert and Suzumura (2018) for the equivalent definition of positional scoring rules.
} 
To prove the "only if" implication $(\Rightarrow)$ of Propositions 1 and 2 it is useful to state the following two Lemmas, proved in the Appendix, that present the scoring rules violating Condorcet consistency for three and five agents.

Lemma 1 Let $n=3$. (a) For any $m \geq 3$, no $w$-scoring rule with $w_{m-2} \in\left(\frac{1}{2}, 1\right]$ is Condorcet consistent. (b) For any $m \geq 5$, no w-scoring rule with $w_{m-2} \in$ $\left[0, \frac{1}{2}\right]$ and $w_{m-3}>w_{1}$, or with $w_{m-2} \in\left[0, \frac{1}{2}\right]$ and $w_{m-2}>w_{m-3}=w_{1}$ is Condorcet consistent.

Lemma 2 Let $n=5$. (a) For $m=3$, no $w$-scoring rule such that $w_{m-2} \in$ $\left[0, \frac{1}{3}\right) \cup\left(\frac{1}{3}, 1\right]$ is Condorcet consistent. (b) For $m \geq 4$, no $w$-scoring rule is Condorcet consistent.

Proof of Proposition 1. The proof of the "only if" implication $(\Rightarrow)$ of both Parts (i) and (ii) is straightforward by Parts (a) and (b) of Lemma 1. It remains to prove the "if" implication of both Parts (i) and (ii). We provide a proof for Part (ii).

(ii) Let $n=3$ and $m=4$. The $w$-scoring rule such that $w_{m-2} \in\left[0, \frac{1}{2}\right]$ is Condorcet consistent. We distinguish the following four cases:

Case 1: $R_{N} \in \mathcal{S}^{n}$ such that $p\left(R_{1}\right)=p\left(R_{2}\right)=p\left(R_{3}\right)=p$. Clearly $C W\left(R_{N}\right)=$ $\{p\}=S_{w}\left(R_{N}\right)$.

Case 2: $R_{N} \in \mathcal{S}^{n}$ such that $p\left(R_{1}\right)=p\left(R_{2}\right)=a_{t}<p\left(R_{3}\right)=a_{t+l}$ for some $t \in\{1, \ldots, m-1\}$ and $l \leqslant m-t$. Note that $C W\left(R_{N}\right)=a_{t}$. Also, observe on the one hand that $s_{w}\left(a_{t}, R_{N}\right) \geq 2 \geq 1+2 w_{m-2}$ since $w_{m-2} \leq \frac{1}{2}$. On the other hand, $s_{w}\left(a_{t+l}, R_{N}\right) \leq 1+2 w_{m-2}$ holds. Moreover, for all $x \notin\left\{a_{t}, a_{t+l}\right\}, s_{w}\left(x, R_{N}\right) \leq$ $3 w_{m-2} \leq \frac{3}{2}$ since $w_{m-2} \leq \frac{1}{2}$. Thus, $s_{w}\left(x, R_{N}\right) \leq \frac{3}{2}<2 \leq s_{w}\left(a_{t}, R_{N}\right)$. Thus, $C W\left(R_{N}\right)=a_{t} \in S_{w}\left(R_{N}\right)$.

Case 3: $R_{N} \in \mathcal{S}^{n}$ such that $p\left(R_{1}\right)=a_{t}<p\left(R_{2}\right)=p\left(R_{3}\right)=a_{t+l}$ for some $t \in\{1, \ldots, m-1\}$ and $l \leqslant m-t$. This case is symmetric to Case 2 exchanging the roles of $a_{t}$ and $a_{t+l}$.

Case 4: $R_{N} \in \mathcal{S}^{n}$ such that $p\left(R_{1}\right)<p\left(R_{2}\right)<p\left(R_{3}\right)$. Note first that $C W\left(R_{N}\right)=$ $p\left(R_{2}\right)$. When computing we obtain that $s_{w}\left(p\left(R_{1}\right), R_{N}\right) \leq 1+w_{m-2}+w_{1}$, $s_{w}\left(p\left(R_{3}\right), R_{N}\right) \leq 1+w_{m-2}+w_{1}$, and $1+w_{m-2}+w_{1} \leq s_{w}\left(p\left(R_{2}\right), R_{N}\right) \leq 1+$ $2 w_{m-2}$. Furthermore, for any alternative $x<p\left(R_{1}\right), s_{w}\left(x, R_{N}\right) \leq s_{w}\left(p\left(R_{1}\right), R_{N}\right)$ and for any alternative $y>p\left(R_{3}\right), s_{w}\left(y, R_{N}\right) \leq s_{w}\left(p\left(R_{3}\right), R_{N}\right)$. Thus, $C W\left(R_{N}\right)=$ $p\left(R_{2}\right) \in S_{w}\left(R_{N}\right)$.

Wrapping up, $S_{w}$ is Condorcet consistent. This shows Part (ii) that can be replicated for Part (i). Thus, the proof ends.

To prove Proposition 2, we need some notation. For $m=3$, there are only four single-peaked preferences, say $R, R^{\prime}, R^{\prime \prime}$, and $R^{\prime \prime \prime}$ defined as follows: $a_{1} R a_{2} R a_{3}, a_{2} R^{\prime} a_{3} R^{\prime} a_{1}, a_{2} R^{\prime \prime} a_{1} R^{\prime \prime} a_{3}$, and $a_{3} R^{\prime \prime \prime} a_{2} R^{\prime \prime \prime} a_{1}$. For any $R_{N} \in \mathcal{S}^{n}$, let $n_{1}$ be the number of agents with preferences $R, n_{2}$ the number of agents with preferences $R^{\prime}, n_{3}$ the number of agents with preferences $R^{\prime \prime}$, and $n_{4} \equiv$ $n-\left(n_{1}+n_{2}+n_{3}\right)$ the number of agents with preferences $R^{\prime \prime \prime}$. Note that the triple $\left(n_{1}, n_{2}, n_{3}\right)$ uniquely defines a profile.

Proof of Proposition 2. Part (ii) of Proposition 2 is proved since it coincides with Part (b) of Lemma 2. To prove Part (i) of Proposition 2, observe that Part 
(a) of Lemma 2 shows the "only if" implication $(\Rightarrow)$ of Part (i). It only remains to prove the "if" implication of Part (i): We have to show that for $n=5$ and $m=3$, the $w$-scoring rule such that $w_{m-2}=\frac{1}{3}$ is Condorcet consistent.

Let $R_{N} \in \mathcal{S}^{n}$ and $\left(n_{1}, n_{2}, n_{3}\right)$ be the triple uniquely associated to $R_{N}$. Since $w_{m-2}=\frac{1}{3}$, then $s_{w}\left(a_{1}, R_{N}\right)=n_{1}+\frac{1}{3} n_{3}, s_{w}\left(a_{2}, R_{N}\right)=\frac{2}{3}\left(n_{2}+n_{3}\right)+\frac{5}{3}$, and $s_{w}\left(a_{3}, R_{N}\right)=n_{4}+\frac{1}{3} n_{2}$. Consider five cases.

Case 1: $n_{1}=5$. Observe that $C W\left(R_{N}\right)=a_{1}$, and $s_{w}\left(a_{1}, R_{N}\right)=5>s_{w}\left(a_{2}, R_{N}\right)=$ $\frac{5}{3}>s_{w}\left(a_{3}, R_{N}\right)=0$ which shows Condorcet consistency.

Case 2: $n_{1}=4$. Observe that $C W\left(R_{N}\right)=a_{1}, s_{w}\left(a_{1}, R_{N}\right) \geq 4, s_{w}\left(a_{3}, R_{N}\right) \leq 1$, and $s_{w}\left(a_{2}, R_{N}\right) \leq \frac{7}{3}$, which shows Condorcet consistency.

Case 3: $n_{1}=3$. Observe that $C W\left(R_{N}\right)=a_{1}$ and that $s_{w}\left(a_{1}, R_{N}\right)=3+\frac{1}{3} n_{3}>$ $s_{w}\left(a_{3}, R_{N}\right)=\left(2-n_{2}-n_{3}\right)+\frac{1}{3} n_{2}=2-n_{3}-\frac{2}{3} n_{2}$. To get Condorcet consistency, it remains to show that $s_{w}\left(a_{1}, R_{N}\right) \geq s_{w}\left(a_{2}, R_{N}\right)$. Since $n=5$ and $n_{1}=3$, then $0 \leq n_{2}+n_{3} \leq 2$, and for any possible such tuple $\left(n_{2}, n_{3}\right)$, we can check that $3+\frac{1}{3} n_{3} \geq \frac{2}{3}\left(n_{2}+n_{3}\right)+\frac{5}{3}$. This ends the proof of Case 3 .

Case 4: $n_{1}=2$. Note that $C W\left(R_{N}\right) \neq a_{1}$. Moreover, $C W\left(R_{N}\right)=a_{3}$ if and only if $n_{4}=3$. The Condorcet consistency of this subcase is proved by symmetry to Case 3 exchanging the roles of $n_{1}$ and $a_{1}$ by $n_{4}$ and $a_{3}$, respectively.

Note that $C W\left(R_{N}\right)=a_{2}$ if and only if $n_{4} \leq 2$, equivalently, $1 \leq n_{2}+n_{3} \leq 3$. Subcase 4.1: $n_{4}=0$, or equivalently, $n_{2}+n_{3}=3$. Note that $s_{w}\left(a_{3}, R_{N}\right)=\frac{1}{3} n_{2}$ $\leq 1$. Moreover, $1<2+\frac{1}{3} n_{3}=s_{w}\left(a_{1}, R_{N}\right)$ and since $n_{3} \leq 3,2+\frac{1}{3} n_{3}<$ $s_{w}\left(a_{2}, R_{N}\right)=2+\frac{5}{3}$. Thus, we get Condorcet consistency.

Subcase 4.2: $n_{4}=1$, or equivalently, $n_{2}+n_{3}=2$. Since $n_{2} \leq 2$, note first that $s_{w}\left(a_{3}, R_{N}\right)=1+\frac{1}{3} n_{2}<1+\frac{2}{3} \leq s_{w}\left(a_{1}, R_{N}\right)=2+\frac{1}{3} n_{3}$. Moreover, since $n_{3} \leq 2$, $s_{w}\left(a_{1}, R_{N}\right)=2+\frac{1}{3} n_{3} \leq 2+\frac{1}{3} 2<3=\frac{2}{3} 2+\frac{5}{3}=s_{w}\left(a_{2}, R_{N}\right)$. Thus, we get Condorcet consistency.

Subcase 4.3: $n_{4}=2$, or equivalently, $n_{2}+n_{3}=1$. Since $n_{3} \leq 1, s_{w}\left(a_{1}, R_{N}\right)=$ $2+\frac{1}{3} n_{3} \leq \frac{7}{3}=s_{w}\left(a_{2}, R_{N}\right)$. Similarly, since $n_{2} \leq 1, s_{w}\left(a_{3}, R_{N}\right)=2+\frac{1}{3} n_{2} \leq$ $\frac{7}{3}=s_{w}\left(a_{2}, R_{N}\right)$. Thus, we get Condorcet consistency.

Case 5: $n_{1}=1$. Note first that $C W\left(R_{N}\right) \neq a_{1}$. Moreover, by definition of $C W$ the following two statements hold: (1) $C W\left(R_{N}\right)=a_{3}$ if and only if $n_{4} \in\{3,4\}$ and (2) $C W\left(R_{N}\right)=a_{2}$ if and only if $n_{4} \leq 2$. We first show Condorcet consistency for situation in (1). Since $n_{4} \in\{3,4\}$, or equivalently $0 \leq n_{2}+n_{3} \leq 1$, $s_{w}\left(a_{3}, R_{N}\right)=n_{4}+\frac{1}{3} n_{2} \geq 3>\frac{7}{3} \geq s_{w}\left(a_{2}, R_{N}\right)=\frac{2}{3}\left(n_{2}+n_{3}\right)+\frac{5}{3}$ and also $s_{w}\left(a_{3}, R_{N}\right)=n_{4}+\frac{1}{3} n_{2} \geq 3>\frac{4}{3} \geq s_{w}\left(a_{1}, R_{N}\right)=1+\frac{1}{3} n_{3}$. To show Condorcet consistency for situation in (2), that is, when $C W\left(R_{N}\right)=a_{2}$, note that $n_{4} \leq 2$ if and only if $2 \leq n_{2}+n_{3} \leq 4$. Observe that $s_{w}\left(a_{1}, R_{N}\right)=n_{1}+\frac{1}{3} n_{3}=1+\frac{1}{3} n_{3}$ is always smaller than $s_{w}\left(a_{2}, R_{N}\right)=\frac{2}{3}\left(n_{2}+n_{3}\right)+\frac{5}{3}$. Note that since $n_{4} \leq 2$, $s_{w}\left(a_{3}, R_{N}\right)=n_{4}+\frac{1}{3} n_{2} \leq 2+\frac{1}{3} n_{2}$ which is smaller or equal than 3 when $n_{2} \leq 3$. Since $2 \leq n_{2}+n_{3}, s_{w}\left(a_{3}, R_{N}\right) \leq 3 \leq s_{w}\left(a_{2}, R_{N}\right)=\frac{2}{3}\left(n_{2}+n_{3}\right)+\frac{5}{3}$. For the case where $n_{2}>3$, that is, $n_{2}=4$, we have that $n_{3}=n_{4}=0$. Clearly, $s_{w}\left(a_{3}, R_{N}\right)=n_{4}+\frac{1}{3} n_{2}=\frac{4}{3}<s_{w}\left(a_{2}, R_{N}\right)=\frac{2}{3}\left(n_{2}+n_{3}\right)+\frac{5}{3}=\frac{13}{3}$. Thus, we prove Condorcet consistency.

Case 6: $n_{1}=0$. Note first that $C W\left(R_{N}\right) \neq a_{1}$ and observe that $s_{w}\left(a_{1}, R_{N}\right)=$ $\frac{1}{3} n_{3}$ is always smaller than $s_{w}\left(a_{2}, R_{N}\right)=\frac{2}{3}\left(n_{2}+n_{3}\right)+\frac{5}{3}$. Moreover, on the one hand, $C W\left(R_{N}\right)=a_{2}$ if and only if $n_{2}+n_{3}>n_{4}$ and $C W\left(R_{N}\right)=a_{3}$ if and only 
if $n_{2}+n_{3}<n_{4}$, since being $n$ odd and $n_{1}=0, n_{2}+n_{3} \neq n_{4}$. On the other hand, the scoring single winner is $a_{2}$, that is, $s_{w}\left(a_{2}, R_{N}\right)=\frac{2}{3}\left(n_{2}+n_{3}\right)+\frac{5}{3}>$ $s_{w}\left(a_{3}, R_{N}\right)=5-n_{3}-\frac{2}{3} n_{2}$ if and only if $4 n_{2}+5 n_{3}>10$, which holds if and only if $n_{2}+n_{3}>n_{4}$. To check the first "if and only if" implication note that $\frac{2}{3}\left(n_{2}+n_{3}\right)+\frac{5}{3}>5-n_{3}-\frac{2}{3} n_{2}$ if and only if $\frac{4}{3} n_{2}+\frac{5}{3} n_{3}-\frac{10}{3}>0$, which holds if and only if $4 n_{2}+5 n_{3}>10$. The latter "if and only if" implication is proved by checking that for each triple $n_{2}, n_{3}$ and $n_{4}$ with $n_{2}+n_{3}>n_{4}$ for the smallest value of $n_{2}+n_{3}$ (that is, 3), the inequality $4 n_{2}+5 n_{3}>10$ holds. Observe that if the latter inequality holds for the smallest value of $n_{2}+n_{3}$, it will also hold for all possible cases. Then, we also have that $C W\left(R_{N}\right)=a_{3}$ if and only if $n_{2}+n_{3}<n_{4}$. We get Condorcet consistency which ends the proof.

Proposition 3 states an impossibility result to obtain Condorcet consistent $w$-scoring rules for seven or more agents. Its proof is in the Appendix.

Summarizing, in this paper, we show that if the size of the group of voters is three, a sub-family of the scoring rules is Condorcet consistent for any number of alternatives. We also show that when there are three alternatives at stake, if the size of the group of voters is between three and five, then some scoring rules are Condorcet consistent.

Lepelley (1996) considers also single-peaked preferences but concentrates on different Condorcet criteria. Moreno and Puy (2005) analyze Condorcet consistency of scoring rules but in a setting where the agenda is not fixed. Bossert and Suzumura (2019) propose a generalization of positional voting rules allowing for non-additive criteria to be included. Skowron, Faliszewski, and Slinko (2019) characterize a class of multi-winner scoring rules satisfying axioms in the spirit of Young's characterization for single-winner scoring methods. In the context of experimental economics, Slater (1958) proposes to estimate the "optimal" group size as the size preferred by group members. The author considers groups of two to seven individuals from a single population who meet several times to discuss about some human relations problem and submit a group solution to it. Slater finds that five-person groups are optimal. Hackman and Vidmar (1970) extend Slater's analysis to the case in which groups members are drawn from two different populations and where three types of intellectual tasks to be carried out by those groups are distinguished. In line with Slater's conclusion, they find that agents in the experiment consider a group size between four and five members as optimal.

\section{Acknowledgements}

We thank the anonymous referee for the extremely detailed remarks on the paper. We also thank A. Nicolò for helpful comments. D. Berga acknowledges the support from the Spanish Ministry of Economy, Industry and Competitiveness through grant ECO2016-76255-P. G. Correa-Lopera and B. Moreno thank the support from the Spanish Ministry of Economy, Industry and Competitiveness through grant ECO2017-86245-P. All authors thank the MOMA network. 


\section{References}

Borda, J.-C. (1781). Mémoire sur les élections au scrutin, Mémoires de l'Académie Royale des Sciences année 1781, 657-665. Translated and reprinted in McLean and Urken (1995, Chapter 5).

Bossert, W. and K. Suzumura (2018). Positionalist Voting Rules: A General Definition and Axiomatic Characterizations. Mimeo.

Condorcet, M.J.A.N. de (1785). Essai sur l'application de l'analyse à la probabilité des décisions rendues à la pluralité des voix, Paris: Imprimerie Royale. Translated and reprinted in part in McLean and Urken (1995, Chapter 6).

Fishburn, P.C. (1973). The Theory of Social Choice, Princeton: Princeton University Press.

Grofman, B., Owen, G. and S.L. Feld (1983). Thirteen theorems in search of the truth. Theory and Decision 15: 261-278.

Hackman, J. R. and N. Vidmar (1970). Effects of Size and Task Type on Group Performance and Member Reactions. Sociometry 33 (1), 37-54.

Lepelley, D. (1996). Constant scoring rules, Condorcet criteria and singlepeaked preferences. Economic Theory 7, 491-500.

May, K. O. (1952). A set of independent necessary and sufficient conditions for simple majority decisions. Econometrica, 20, 680-684.

Moreno, B. and S. Puy (2005). The scoring rules in an endogenous election. Social Choice and Welfare 25, 115-125.

Moulin, H. (1988). Axioms of Cooperative Decision Making. Econometric Society Monographs No. 15. Cambridge University Press.

Skowron, P., P. Faliszewski, and A. Slinko (2019). Axiomatic characterization of committee scoring rules. Journal of Economic Theory 180, 244-273.

Slater, P. E. (1958). Contrasting Correlates of Group Size. Sociometry 21 (2), 129-139.

Smith (1973). Aggregation of preferences with variable electorate. Econometrica 41, 1027-1041.

Young, H.P. (1975). Social choice scoring functions. SIAM Journal on Applied Mathematics 28, 824-838.

\section{Appendix}

We prove Proposition 3 and Lemmas 1 and 2 used for the proof of Propositions 1 and 2. We first introduce and prove six Lemmas.

Define four preferences used in the proofs of Lemmas 3 to $7: R \in \mathcal{S}$ is such that $a_{1} R a_{2} R \ldots R a_{m-1} R a_{m}, R^{\prime} \in \mathcal{S}$ is such that $a_{2} R^{\prime} a_{3} R^{\prime} \ldots R^{\prime} a_{m} R^{\prime} a_{1}$, $R^{\prime \prime} \in \mathcal{S}$ is such that $a_{m} R^{\prime \prime} a_{m-1} R^{\prime \prime} \ldots R^{\prime \prime} a_{2} R^{\prime \prime} a_{1}$, and $R^{\prime \prime \prime} \in \mathcal{S}$ is such that $a_{3} R^{\prime \prime \prime} a_{4} R^{\prime \prime \prime} a_{5} R^{\prime \prime \prime} \ldots R^{\prime \prime \prime} a_{m} R^{\prime \prime \prime} a_{2} R^{\prime \prime \prime} a_{1}$. To prove these lemmas, we propose preference profiles for which Condorcet consistency fails.

Lemma 3 For any $n \geq 3$ and $m \geq 3$, no w-scoring rule with $w_{m-2} \in\left(\frac{1}{2}, 1\right]$ is Condorcet consistent.

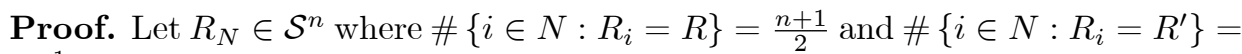
$\frac{n-1}{2}$. Note that $C W\left(R_{N}\right)=a_{1}$ and $x \notin S_{w}\left(R_{N}\right)^{2}$ for $x \in A \backslash\left\{a_{1}, a_{2}\right\}$ since 
$s_{w}\left(a_{2}, R_{N}\right)>s_{w}\left(x, R_{N}\right)$. Moreover, $s_{w}\left(a_{2}, R_{N}\right)=\frac{n-1}{2}+\frac{n+1}{2} w_{m-2}>\frac{n-1}{2}+\frac{n+1}{4}$ and $\frac{n+1}{2}+\frac{n-3}{4} \geq \frac{n+1}{2}=s_{w}\left(a_{1}, R_{N}\right)$ since $n \geq 3$. Thus, $S_{w}\left(R_{N}\right)=\left\{a_{2}\right\}$.

Lemma 4 For any $n \geq 5$ and $m \geq 4$, no w-scoring rule with $w_{m-2}<1$ is Condorcet consistent.

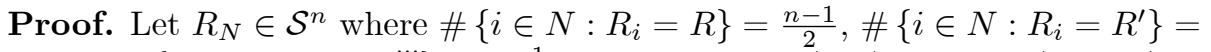
1 , and \# $\left\{i \in N: R_{i}=R^{\prime \prime \prime}\right\}=\frac{n-1}{2}$. Note that $C W\left(R_{N}\right)=a_{2}, s_{w}\left(a_{2}, R_{N}\right)=$ $\frac{n-1}{2} w_{m-2}+1+\frac{n-1}{2} w_{1}$, and $s_{w}\left(a_{3}, R_{N}\right)=\frac{n-1}{2}+w_{m-2}+\frac{n-1}{2} w_{m-3}$. Since $\frac{n-1}{2}=$ $1+\frac{n-3}{2}$ and $w_{m-2}<1$, we obtain that $s_{w}\left(a_{3}, R_{N}\right)>\frac{n-1}{2} w_{m-2}+1+\frac{n-1}{2} w_{m-3}$. Moreover, $\frac{n-1}{2} w_{m-2}+1+\frac{n-1}{2} w_{m-3} \geq s_{w}\left(a_{2}, R_{N}\right)$ since $w_{m-3} \geq w_{1}$. Thus, $a_{2}=C W\left(R_{N}\right) \notin S_{w}\left(R_{N}\right)$.

Lemma 5 For any $n>5$ and $m=3$, no $w$-scoring rule with $w_{m-2} \in\left[0, \frac{1}{2}\right]$ is Condorcet consistent.

Proof. Distinguish three cases: (1) $w_{m-2}<\frac{n-3}{2(n-2)},(2) w_{m-2}>\frac{n-3}{2(n-2)}$, and (3) $w_{m-2}=\frac{n-3}{2(n-2)}$.

Case 1: let $R_{N} \in \mathcal{S}^{n}$ such that $\#\left\{i \in N: R_{i}=R\right\}=\frac{n-1}{2}, \#\left\{i \in N: R_{i}=R^{\prime}\right\}=$ 1 , and \# $\left\{i \in N: R_{i}=R^{\prime \prime}\right\}=\frac{n-1}{2}$. Note that $C W\left(R_{N}\right)=a_{2}$. We can check that $s_{w}\left(a_{2}, R_{N}\right)=(n-1) w_{m-2}+1, s_{w}\left(a_{3}, R_{N}\right)=w_{m-2}+\frac{n-1}{2}$, and thus, $s_{w}\left(a_{3}, R_{N}\right)>s_{w}\left(a_{2}, R_{N}\right)$ if and only if $w_{m-2}<\frac{n-3}{2(n-2)}$.

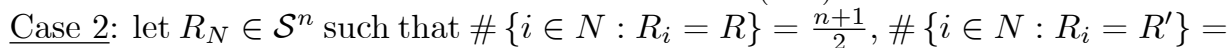
2, and $\#\left\{i \in N: R_{i}=R^{\prime \prime}\right\}=\frac{n-5}{2}$. Note that $C W\left(R_{N}\right)=a_{1}$. We can check that $s_{w}\left(a_{2}, R_{N}\right)=(n-2) w_{m-2}+2, s_{w}\left(a_{1}, R_{N}\right)=\frac{n+1}{2}$, and thus, $s_{w}\left(a_{2}, R_{N}\right)>s_{w}\left(a_{1}, R_{N}\right)$ if and only if $w_{m-2}>\frac{n-3}{2(n-2)}$.

Case 3: let $R_{N} \in \mathcal{S}^{n}$ such that $\#\left\{i \in N: R_{i}=R\right\}=\frac{n+1}{2}, \#\left\{i \in N: R_{i}=R^{\prime}\right\}=$ 3, and $\#\left\{i \in N: R_{i}=R^{\prime \prime}\right\}=\frac{n-7}{2}$. Note that the profiles is well-defined since $n>5$. Note that $C W\left(R_{N}\right)=a_{1}$. We can check that $s_{w}\left(a_{2}, R_{N}\right)=$ $(n-3) w_{m-2}+3, s_{w}\left(a_{1}, R_{N}\right)=\frac{n+1}{2}$, and thus, $s_{w}\left(a_{3}, R_{N}\right)>s_{w}\left(a_{1}, R_{N}\right)$ if and only if $w_{m-2}>\frac{n-5}{2(n-3)}$, which holds for all $n>1$, since $w_{m-2}=\frac{n-3}{2(n-2)}$.

Lemma 6 For any $n=5$ and $m=3$, no $w$-scoring rule with $w_{m-2} \in\left[0, \frac{1}{3}\right) \cup$ $\left(\frac{1}{3}, \frac{1}{2}\right]$ is Condorcet consistent.

The profiles of preferences in Cases (1) and (2) in the proof of Lemma 5 work to prove Lemma 6.

Lemma 7 For any $n=3$ and $m \geq 5$, no $w$-scoring rule with $w_{m-2} \in\left(0, \frac{1}{2}\right]$ and $w_{m-3}>w_{1}$ is Condorcet consistent.

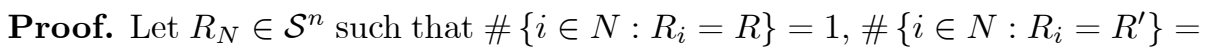
1 , and \# $\left.\# i \in N: R_{i}=R^{\prime \prime}\right\}=1$. Note that $C W\left(R_{N}\right)=a_{2}$. We can check that $x \notin S_{w}\left(R_{N}\right)$ for $x \in A \backslash\left\{a_{1}, a_{2}, a_{3}\right\}$ since $s_{w}\left(a_{2}, R_{N}\right)>s_{w}\left(x, R_{N}\right)$. Moreover, since $s_{w}\left(a_{2}, R_{N}\right)=1+w_{m-2}+w_{1}<s_{w}\left(a_{3}, R_{N}\right)=1+w_{m-2}+w_{m-3}$ by $w_{m-3}>w_{1}$, we get that $C W\left(R_{N}\right)=a_{2} \notin S_{w}\left(R_{N}\right)$. 
Lemma 8 For any $n=3$ and $m \geq 5$, no $w$-scoring rule with $w_{m-2} \in\left[0, \frac{1}{2}\right]$ and $w_{m-2}>w_{m-3}=w_{1}$ is Condorcet consistent.

Proof. Take any $R_{N} \in \mathcal{S}^{3}$ such that the preferences of the three agents are $\widetilde{R}, \bar{R}$, and $\widehat{R} \in \mathcal{S}$, where $a_{2} \widetilde{R} a_{1} \widetilde{R} a_{3} \widetilde{R} \ldots \widetilde{R} a_{m-1} \widetilde{R} a_{m}, a_{3} \bar{R} a_{2} \bar{R} a_{4} \bar{R} a_{1} \bar{R} a_{5} \bar{R} \ldots \bar{R} a_{m}$, and $a_{4} \widehat{R} a_{5} \widehat{R} a_{3} \widehat{R} a_{2} \widehat{R} a_{1} \widehat{R} \ldots \widehat{R} a_{m}$. Note that $C W\left(R_{N}\right)=a_{3}$. We can check that $x \notin S_{w}\left(R_{N}\right)$ for $x \in A \backslash\left\{a_{2}, a_{3}, a_{4}\right\}$ since $s_{w}\left(a_{2}, R_{N}\right)=1+w_{m-2}+w_{1}>w_{m-2}+$ $2 w_{1} \geq s_{w}\left(x, R_{N}\right)$. Moreover, $s_{w}\left(a_{2}, R_{N}\right)=1+w_{m-2}+w_{1}>s_{w}\left(a_{3}, R_{N}\right)=$ $s_{w}\left(a_{4}, R_{N}\right)=1+2 w_{1}$ since $w_{m-2}>w_{1}$. Then, we get that $C W\left(R_{N}\right)=a_{3} \notin$ $S_{w}\left(R_{N}\right)=\left\{a_{2}\right\}$.

The proof of Lemma 1 is straightforward by Lemmas 3, 7, and 8 .

The proof of Lemma 2 is straightforward by Lemmas 3, 4, and 6 .

The proof of Proposition 3 is straightforward by Lemmas 3 , 4, and 5 . 